\title{
Claude Fintz
}

\section{Entre emprise et déprise :}

\section{I'imnginaire croisé de l'altérité entre embrigadeurs et désembrigadeurs}

BETWEEN INFLUENCE AND UN-INFLUENCE:

THE CROSSED IMAGINATION OF OTHERNESS, SHARED BY PEOPLE WHO INDOCTRINATE AND BY PEOPLE WHO UNDO INDOCTRINATION Abstract: We can easily be taken in by the illusion that otherness, in the present context, builds itself on an opposition to the values of the West that rejects violently the one who lets himself be indoctrinated. But otherness is not only an ideological and religious construction: it is sometimes also an answer to the internal faintness of the young person; establishing at the same time a barrier against Evil and personal anxiety, it no longer concerns otherness, but a sort of selfindoctrination.

Keywords: Otherness; (Self)indoctrination; Community of Imagination; Opposition; Faiths.

\section{Claude FinTZ}

Université Grenoble Alpes, France claude.fintz@univ-grenoble-alpes.fr

DOI: $10.24193 /$ cechinox.2019.36.08
Lextériorité se constate, l'altérité se construit. François Jullien

Je suis certain aujourd'hui que la radicalité des jeunes gens que j'ai rencontrés résulte de la difficulté grandissante de nos sociétés à intégrer la différence - non pas celle du

« semblable » dont on nous rebat les oreilles, mais de l'autre, vraiment autre, radicalement autre. Les âmes mortes, Tobie Nathan, L'iconoclaste, 2017, p. 242.

$\mathrm{D}$ ans la présente étude qui concerne les jeunes Français qui partent pour la Syrie, j'ai souhaité observer le travail imaginaire dans l'espace sociétal, abandonnant provisoirement celui de l'œuvre littéraire ou esthétique, au profit de l'œuvre sociétale. Voici un sujet délicat que je souhaite aborder avec précaution et neutralité car, dans cet essai, la pensée se construit de façon expérimentale.

Pour traiter mon sujet, je m'appuierai sur le point de vue exposé dans les ouvrages 
de Dounia Bouzar ; dans ceci, on constate que le récit idéologique proposé se fonde sur un imaginaire dichotomique de l'altérité, que chaque camp se renvoie de façon croisée. Je ne m'intéresserai ici qu’à un aspect d'un phénomène complexe et qu'à un profil, très spécifique, de candidat : un jeune, une «âme errante » (Tobie Nathan), parfois enfant de migrant qui, après endoctrinement, adopte le costume de l'élu pour rétablir la justice et la vérité sur le monde.

Les imaginaires du corps se prêtent idéalement à l'expression de l'altérité car ils symbolisent l'appartenance à une entité constituée. Faire corps revient à agréger le même au même et constitue a priori un symbole de la fermeture, qui pense l'autre comme altérité : on fait corps avec mais aussi contre. Mais s'agissant, comme ici, d'univers de croyances qui empruntent les uns aux autres, les deux univers, contrairement à toute attente, ne seraient-ils pas en train de construire du commun et de se référer à une même série d'imaginaires ?

\section{Le mécanisme structurel de l'altérité dichotomique}

Commençons par analyser les deux pôles présentés comme antagonistes. Ce singulier embrigadement se donne comme une lutte contre l'idéologie d'un adversaire. On est en présence de deux paradigmes en rupture radicale, laquelle se marque par une opposition axiologique.

Il existe ainsi une " guerre des dieux " (Tobie Nathan) où, pour les " engagés ", notre monde est en dilution, en déshérence, voire en décadence ; à celui-ci, ils opposent un univers tendu vers un horizon épique, utopique, porté par la mythologie de la régénération, elle-même portée par un horizon eschatologique. La caution divine est censée autoriser provisoirement désordre et ubris afin d'introduire un nouvel ordre, où cesseront les transgressions.

On trouve l'opposition d'un monde profondément désenchanté, dont le merveilleux religieux a été évacué, où le récit social (et national) est exsangue, et de l'autre un monde ré-enchanté sur lequel on se (re)branche. Dans ce monde parfaitement régulé, le désespoir de la pensée solitaire est évacué par la saturation ritualisée du collectif. D'un côté, se trouve un univers terne, atone, où règne l'absence de tension autre que celle de la consommation frénétique ; de l'autre, on a une pensée tragique, qui induit de puissantes émotions communautaires, articulées sur des croyances religieuses, une épopée planétaire.

Comme dans tout récit bien scénarisé, se dessine un système d'opposition de valeurs. On développe sur le net, dans les vidéos de propagande, une rhétorique du diable et des pratiques sataniques, en lien avec l'ennemi désigné : les sociétés secrètes ou l'État d'Israël. Chacun fait de l'autre un barbare : on voit d'un côté le massacre des enfants, des femmes et des vieillards syriens ; quant aux médias occidentaux, ils ne donnent des autres que le spectacle d'actes de cruauté insignes et d'anti-civilisation (décapitations, destruction de Palmyre).

Par ailleurs, on persuade l'embrigadé que l'altérité, c'est le monde social et familial dans lequel il a baigné jusque-là, qui symbolise l'univers du mensonge, de l'endoctrinement et de l'ignorance. Du mensonge à la vérité, de la perdition à l'élection, de l'ignorant qu'il était, au "véridique " qu'il est devenu : voilà l'itinéraire qu'il est proposé au jeune d'incarner. 
Lautre (y compris son semblable) prend petit à petit la figure du mal sans visage ; on le néantise symboliquement, pour parvenir ensuite à le détruire sans état d'âme. D'un côté, on trouve le monde de la perdition, dont on doit à toute force retirer soi-même et les siens; de l'autre, la Terre pure, la terre de Cham ; plus lointain, après le combat et la mort, est déployé l'espace céleste où les combattants seront récompensés. Enfin la reprise du monde par le califat est envisagée comme le retour du paradis sur terre.

Le système d'emprise se matérialise à travers la création d'une bulle virtuelle, entretenue par les vidéos et le contact étroit d'un guide et de l'impétrant par Internet ; le blindage symbolique (protections symboliques - vestimentaires et rituelles, pour se tenir à distance de l'entité contaminée) induit un "faire corps » communiel, coupant radicalement le sujet avec l'ordre ancien. Dans ce chemin d'initiation, le myste est amené à s'amputer progressivement de son « identité » antérieure. En ce sens, l'initiation sectaire est bien un processus d'altérisation.

Par ailleurs, les idéologues promeuvent l'importance des épreuves pour s'extraire du monde matérialiste. Le binarisme radical de Dieu et du diable, est très pratique pour constituer en adversaires des univers paranoïdes. Ce binarisme s'accompagne d'un processus de diabolisation de l'autre par le biais de la pensée religieuse, qui devient caution de l'idéologie. Ainsi l'univers occidental est présenté comme un adversaire occulte : les Illuminati sont convoqués sur une rive ; la hantise d'un projet d'asservissement mondial sur l'autre. Complotisme et paranoïa s'entretiennent mutuellement et présupposent l'existence d'un univers dichotomique, reflétant le même projet concerté de destruction de l'autre.

L'altérité est bien ici une construction discursive et idéologique. Maisla dualité se réverbère du dehors vers le dedans - et l'adversaire, présenté comme externe, s'avère être autant interne : l'autre de soi, l'alter ego.

\section{Une lutte contre l'autre de soi, une barrière contre le mal et l'angoisse}

T 'engagé est exposé à l'idée que l'autre (famille, modes de vie et culture) étant en soi, une partie de soi, il faut donc l'amputer. Première coupure, première division. Servant de rempart contre l'autre de soi les agressions intérieures / extérieures, la pensée sectaire est ressentie comme une barrière et une protection contre le mal. Elle explique, dans la logique d'opposition, lexistence des forces du mal, contre lesquelles la communauté des véridiques se déclare prête à se sacrifier. Le diable, l'adversaire, convoqué à tout propos, veut faire échouer le candidat à l'élection, puisqu'il est censé pousser ce dernier vers l'abandon et la facilité, quand les difficultés seraient la manifestation même de l'épreuve divine.

Mais - autre et très grave division intérieure - le candidat est conscient d'être en partie responsable de sa propre aliénation: " tu t'en doctrines toi-même en fait. On est tous nos propres gourous. On est tous à la fois victimes et coupables $»^{1}$. Inversement, c'est a posteriori, lors des entretiens avec le groupe d'intervention de DB, que le jeune parvient à prendre du recul par rapport à l'illusion auto-construite - et à se dégager de l'emprise objective / subjective. L'emprise, c'est lui-même qui l'alimentait, à 
partir de motifs personnels cachés. Mais si, malgré ses doutes, l'embrigadé reste soumis à la parole donnée, c'est pour ne pas avoir à renier des choix antérieurs : le mécanisme d'enfermement est parfait. S'étant rendu dans l'autre camp, on n'a plus accès à soi. On est pris dans une illusion d'altérité - car l'embrigadeur, ultimement, c'est soi-même.

De l'autre bord, on voit l'embrigadé comme un psychopathe relevant d'une communauté non humaine. Il s'est altérisé : sa personnalité et son jugement ont été digérés par le groupe cannibale ou vampire : «Ali a sombré : le groupe l'a mangé »². Le dogme sectaire, qui unifie le groupe, semble être devenu l'adversaire véritable. Ainsi le combattant piégé, engagé dans des actes criminels qu'il ne pensait pas pouvoir ni devoir commettre, à son retour en France, n'a plus de repères (puisqu'ils ont à deux fois explosé). Troisième schize : il ne se reconnaît plus dans aucune identité ; l'altérité est désormais partout.

C'est pourquoi le groupe d'intervention de Dounia Bouzar tente de faire ré-émerger sa pensée critique et éthique ; pour cela, il tente de susciter le retour de la mémoire affective du jeune, le faisant sortir de l'insensibilité émotionnelle où il s'était enfermé. Ainsi chacune des deux postures, étroitement liée à l'autre, fait semblant d'ignorer qu'elle est elle-même une partie de l'autre. Comment s'effectuent ces inversions, où l'altérité s'avère être la face cachée de l'identité ?

\section{Croisements, inversions, emprunts, transferts autour de l'altérisation}

$\mathrm{U}$ ne première inversion se constate dans le sentiment négatif que chacun a de la position de l'autre : celui qui se croit éveillé voit sa famille et ses amis plongés dans le sommeil de l'ignorance ; à l'inverse, « le véridique " apparaît comme un aliéné frappé de stupeur : donc, de ce point de vue, la radicalisation serait tout le contraire de léveil qu'il croyait avoir atteint. Là où les embrigadés sont sûrs de se déployer spirituellement, les seconds sont persuadés que les premiers ont subi une mutilation d'euxmêmes, puisque par leur conversion, ils se sont radicalement coupés de leurs racines culturelles et sociales.

Le second point de jonction concerne les embrigadeurs qui utilisent toutes les techniques de marketing occidentales, exploitant toute la rhétorique du discours et des images. On séduit la population que l'on cherche à détourner en utilisant comme armes les technologies médiatiques, qui constituent le moteur de l'imaginaire de la jeunesse occidentale : ici on utilise les technologies pour transformer le même en autre. Des analyses ont détecté, dans les vidéos de "propagande ", des techniques de scénarisation ultrasophistiquées que seuls des étudiants occidentaux ont pu acquérir, ce qui suppose d'avoir détourné ses armes contre l'Occident (« butin de guerre » de Kateb Yacine, à propos de la langue française). Par les emprunts faits au pôle adverse, on détourne ainsi l'autre par le même.

À la croisée des deux pôles antagonistes se trouve une troisième notion stratégique : celle d'engagement, présente en miroir, à "l'aller et au retour ». C'est précisément sur le mode de l'emprunt réciproque que l'on propose, comme le fait l'équipe de $\mathrm{DB}$, au jeune qui tente de se désengager du lien sectaire pour se ré-engager sur la chaîne sociétale. En effet, pour s'engager de façon radicale, il lui a fallu se 
dégager de l'engagement antérieur. Inversement, lorsque, déçu et désorienté, il revient de Syrie en France, on lui demande d'aider à désengager ceux qui sont encore sous emprise d'un engagement, dont il essaie précisément lui-même de se séparer. Traitement homéopathique, du même par le même. L'engagement est simplement inversé - ce qui souligne l'identité des procédures prétendument « opposées ».

Cette phase d'inversion et d'emprunts mutuels tend à souligner l'inexistence d'une véritable opposition dans les pôles considérés comme relevant a priori d'une altérité intrinsèque. C'est pourquoi je vais souligner à présent l'existence d'une zone d'ambiguité et de confusion : zone de raccord, de coupure / lien entre les deux mondes.

\section{La zone interlope et (con)- fusionnelle du travail imaginaire}

$\mathrm{U}_{\mathrm{s}, \mathrm{m}}$

ne phase de régression avait impliqué le sujet embrigadé dans une corporéité groupale, une bulle virtuelle et émotionnelle, dans laquelle la (con)fusion lui conférait un sentiment d'assurance, de protection et d'exaltation collective. Au retour, la régression s'est métamorphosée en dépression.

Ainsi, le jeune se trouve sans cesse dans un inter-monde, un Bardo qui correspond à un état émotionnel, à mi-chemin entre l'exaltation et le coma. Il vit dans un monde à part : le monde alternatif des véridiques, a priori sans retour vers la vie sociale (pour le prouver, on voit des jeunes brûler leur passeport). De même, à son retour, après avoir subi de graves désillusions ou d'intenses traumatismes, il retourne dans un inter-monde : il ne parvient plus à coïncider avec lui-même, son identité psycho-spirituelle ayant été fracturée à deux reprises. Il appartient à un no man's land terrifiant, une sorte d'enfer - puisqu'il ne peut plus croire ni en les siens, ni dans les autres, ni en lui-même.

Les expressions de l'altérité sont donc bien une construction qui correspond à une logique binaire, fondé sur le principe de contradiction, qui apparait soudain comme totalement instable. En effet, soutenant cette logique du tiers inclus, une série d'imaginaires paraissent communs aux deux pôles de l'altérité.

J'en envisagerai cinq :

1. Limaginaire du jour, de la nuit reprend l'opposition mensonge / vérité, identité / altérité. En fait, chacun se trouvant dans un entre-monde où l'opposition du jour et de la nuit n'est pas tranchée, elle donne naissance à l'imaginaire crépusculaire, espace indéterminé ou confusionnel, de qui se cherche, de qui est perdu ou de qui s'est fondu dans un corps de groupe.

2. Limaginaire de la manipulation : travail de la " main » mentale qui agit magiquement sur l'autre, dans le sens d'un faire croire et qui exerce une emprise sur l'autre ; chacun manipule et inversement, est manipulé, voire se manipule.

3. L'imaginaire de la contamination: elle symbolise le mal transmis par l'autre, sciemment ou à son corps défendant; la pensée et la croyance sont de nature virale, et communiquer avec l'autre signifie qu'on puisse vouloir le contaminer : faire du prosélytisme (l'aliéner) n'est donc, en soi, pas un mal : " C'est faire du bien aux autres que de les endoctriner ${ }^{3}$.

4. L'imaginaire de la dévoration, de l'incorporation et de la fusion : "Ali a 
sombré : le groupe l'a mangé». L'envers de la dévoration est la fusion émotionnelle, autoalimentée, puis nostalgiquement regrettée au retour. Lextase collective, expression d'une corporéité mystique, fonctionne de conserve avec son double : la dévoration, l'incorporation cannibale. Accepter de faire partie de la chaîne revient à être incorporé.

5. L'imaginaire de la chaîne et / ou du lien (solidarité et / ou aliénation) : il existe une zone indécise où les postures conflictuelles se résorbent et s'inversent.

Dans les deux situations, demeure la chaîne qui permet de (re)faire lien ; ce lien émotionnel, activé à l'aller dans un environnement tragique et réactivé au voyage-retour, raccorde l'archaïque à la modernité. Il soude deux mondes, par une paradoxale coupure / lien.

La rencontre d'une mythologie collective et la construction d'un mythe personnel sont le lieu du travail imaginaire, où le sujet métamorphose un destin social insatisfaisant en la quête d'un héroïsme accompli, le faisant pleinement entrer dans le mythe. Cependant, la soumission n'est possible que s'il y a acceptation du jeune à avancer de lui-même vers une altérité qui lui est révélée comme étant son identité profonde. L'aliénation provient dès lors du sujet lui-même qui renforce la contrainte de la chaîne qu'il s'est mise autour du cou.

\section{Conclusion}

L e régime de l'imaginaire crépusculaire, contigu à celui de la mort, désigne une zone interlope, un bardo, un entre monde où l'identité et l'altérité se mélangent, mettant en lumière une perspective où les opposés se ressemblent et s'annulent car l'altérité se nourrit de l'auto-illusion.

Un imaginaire complexe est convoqué, qui passe par les figures de la contamination virale, de la manipulation (réciproque), de la fusion émotionnelle, de la dévoration, du lien et de la chaîne. Cet imaginaire, déconnecté d'un univers polarisé et dualiste, de par sa fondamentale ambiguité, délimite une zone d'incertitude, d'indétermination, où le même peut générer l'autre, selon les choix d'orientation que prennent le sujet ou l'idéologie.

Se pose alors la question suivante : la radicalisation, qui est un engagement dans des croyances, est-elle absolument d'une autre nature que celle à laquelle nous adhérons socialement, par addiction consensuelle ? Par un effet d'aller-retour du regard, on voit apparaitre, du reste, la violence symbolique intrinsèque à tout pacte social, et du nôtre en particulier. C'est sans doute pourquoi l'affiliation à un credo sectaire, qui déresponsabilise le sujet, est vécue comme une libération, un miraculeux allégement du poids de la culture et de sa personne.

Plus profondément encore, y aurait-il, comme le pense Tobie Nathan, enfouie sous la surface des représentations sociales, " une guerre des dieux », toujours latente et prête à se réactiver, que la parole des jeunes radicalisés aurait le mérite de mettre en avant ? Voici pour finir les propos étonnamment prémonitoires, que Tobie Nathan reprend, citant Max Weber :

La multitude des dieux antiques sortent de leur tombe, sous la forme de puissances impersonnelles, parce que désenchantées, et ils s'efforcent à 
L'imaginaire croisé de l'altérité entre embrigadeurs et désembrigadeurs

nouveau de faire retomber notre vie en leur pouvoir tout en reprenant leurs luttes éternelles. D'où les tourments de l'homme moderne qui se révèlent tout particulièrement pénibles pour la jeune génération (Weber).

\section{Bibliographie}

Bouzar, Dounia, Comment sortir de l'emprise jihadiste, Ivry-sur-Seine, Les Éditions de l'atelier, 2015.

- Ils cherchent le paradis, ils ont trouvé l'enfer, Ivry-sur-Seine, Les Éditions de l'atelier, 2014.

- La vie après Daesh, Ivry-sur-Seine, Les Éditions de l'atelier, 2015.

- Ma meilleure amie s'est fait embrigader, Paris, Éditions De la Martinière, 2016.

Bouzar, Douna, Farid Benyettou, Mon jihad, itinéraire d'un repenti, Paris, Éditions Autrement, 2017.

Nathan, Tobie, Les âmes mortes, Paris, L'iconoclaste, 2017.

Thomson, David, Les Revenants, Paris, Points Doc, 2018.

Weber, Max, Le savant et le politique, Paris, 10 / 18, 1963.

\section{Filmographie}

Et le ciel attendra, Marie-Castille Mention-Schaar, 2016.

\section{Notes}

1. Dounia Bouzar, Ma meilleure amie s'est fait embrigader, Paris, Éditions De la Martinière, 2016, p. 193.

2. Ibid., p. 98.

3. Ibid., p. 177. 